\title{
Correlation of Graft Maturity with Knee Function Scores Post Anterior Cruciate Ligament Reconstruction: A Retrospective Study
}

Rongjin Chen ( $\nabla$ cgxygraj@163.com )

Chengdu University of Traditional Chinese Medicine Wenjiang Campus: Chengdu University of

Traditional Chinese Medicine

\section{Zhu Haozhong}

Affiliated Chengdu Fifth people's Hospital of Chengdu University of Traditional Chinese Medicine

Gu Xinyi

Affiliated Chengdu Fifth people's Hospital of Chengdu University of Traditional Chinese Medicine xiang xianxiang

Affiliated Zhongshan Hospital of Dalian University

\section{Research article}

Keywords: Anterior Cruciate Ligament Reconstruction, Femur, Magnetic Resonance Imaging, Retrospective Studies, Tendons, Tibia

Posted Date: September 17th, 2021

DOI: https://doi.org/10.21203/rs.3.rs-898511/v1

License: (c) (1) This work is licensed under a Creative Commons Attribution 4.0 International License. Read Full License 


\section{Abstract}

BACKGROUND: The relationship between the signal/noise quotient value and knee function scores has not been extensively studied. Thus, we aimed to investigate the correlation between graft maturity and knee function after anterior cruciate ligament reconstruction.

METHODS: In this retrospective study, we included patients ( $n=50 ; 28$ male patients; age, 31.0 years) who underwent anterior cruciate ligament reconstruction with autogenous tendons between August 2016 and August 2018. At 6 months and 2 years postoperatively, the signal/noise quotient values of the tibia and femur, indicating graft maturity, were measured using magnetic resonance imaging. The Tegner, Lysholm, and International Knee Documentation Committee scores were used to evaluate knee function. Mean signal/noise quotient values of the grafts' femoral and tibial ends were considered the final signal/noise quotient values. The correlation between the signal/noise quotient value at 6 months postoperatively and knee function score at 2 years postoperatively was analyzed.

RESULTS: All patients were followed up for 24-28 months. The International Knee Documentation Committee, Lysholm, and Tegner scores of the knee joint at 6 months and 2 years postoperatively were significantly higher than the preoperative scores, and those at 2 years postoperatively were significantly higher than those at 6 months postoperatively. The signal/noise quotient value for all patients at 6 months postoperatively was negatively correlated with the Lysholm, International Knee Documentation Committee, and Tegner scores at 2 years postoperatively.

CONCLUSION: The signal/noise quotient value based on magnetic resonance imaging in the early stage after anterior cruciate ligament reconstruction can predict knee function in the later stage.

\section{Background}

Arthroscopic reconstruction is an effective way to treat anterior cruciate ligament (ACL) injury. The main goal is to restore stability and improve knee joint function as much as possible [1, 2]. The Tegner, Lysholm, and International Knee Documentation Committee (IKDC) scores are important evaluation indices of knee joint function after ACL reconstruction [3-5].

Weiler et al. [6] found that the signal/noise quotient (SNQ) based on magnetic resonance imaging (MRI) can well display the vascularization and ligamentization process of grafts and revealed that graft maturity (SNQ value) was negatively correlated with the maximum failure load, tensile strength, and stiffness of grafts $[7,8]$.

However, currently, there are only a few studies on the relationship between the SNQ value and knee function scores. Li et al. [9] reported that MRI-based ACL graft maturity does not predict clinical and functional outcomes during the first year after ACL reconstruction. However, Li et al. [5] demonstrated that early measurement of graft maturity can predict clinical outcomes after ACL reconstruction, and the SNQ can be used for the determination of the prognosis of patients. Despite some positive findings in graft 
maturation and knee function scores, further studies are needed to determine whether the SNQ value based on MRI can be used to predict knee function. Therefore, we retrospectively analyzed the clinical data of patients undergoing ACL. We explored the correlation between graft maturity and knee function after ACL reconstruction to guide the functional exercise of patients according to the graft maturity after reconstruction. This may change the current subjective characteristics of functional exercise guided by clinical experience, ensure the safety and feasibility of rehabilitation exercise to the greatest extent, improve the success rate of ACL reconstruction, and provide an objective basis to evaluate ACL reconstruction and rehabilitation outcomes. This study was conducted in accordance with the principles of the Declaration of Helsinki and was approved by the ethics committee of our institution (No. 2019172). All study participants provided informed consent.

\section{Methods}

The present study included patients who underwent $A C L$ reconstruction using autogenous tendons at our institute from August 2016 to August 2018. The inclusion criteria were as follows: a diagnosis of ACL rupture using preoperative imaging and physical examination; age, 18-50 years; unilateral ACL single bundle reconstruction performed for the first time; and postoperative rehabilitation training and follow-up performed by doctors. The exclusion criteria were knee joints with severe osteoarthritis (Kellgren Lawrence grade $\geq \mathrm{III}$ ); confirmation of meniscal injury on MRI or arthroscopic exploration and suture requirement in addition to operation; confirmation of cartilage injury on arthroscopic exploration (Outerbridge grade $\geq \mathrm{III}$ ); and multiple ligament injury and infection.

Surgery was performed under general or epidural anesthesia. The patients were placed in the supine position; the lithotomy position was adopted for the healthy knee, while the affected knee drooped naturally. After ACL rupture was confirmed by anteromedial and anterolateral arthroscopic approaches, a 3 -cm longitudinal skin incision was made approximately $2 \mathrm{~cm}$ medial to the tibial tubercle. Parts of the semitendinosus and gracilis tendons were cut and woven to prepare the tendon graft, with a diameter of 6-8 $\mathrm{mm}$ and length of $9-10 \mathrm{~cm}$. According to the diameter of the graft, tibial and femoral tunnels were drilled into the tibial and femoral anatomical footprints of the ACL. The graft was pulled from the tibial tunnel to the femoral tunnel using the traction line, and the femoral end was fixed with an adjustable suspension titanium plate (DePuy Mitek Surgical Products, Inc. Raynham, MA, USA). The tibial end of the graft was then pulled with 20-N tension, and the joint was repeatedly flexed and extended 20 times. After confirming that there was no contact between the graft and the intercondylar fossa under arthroscopy, the tibial end was fixed with a compression screw with the knee in the extended position (DePuy Mitek Surgical Products, Inc. Raynham, MA, USA). The incision was closed; and the tension and fixation of the graft were reexplored.

After anesthesia recovery, the patients performed straight leg raises and ankle pump exercises in bed. Twenty-four hours later, they wore a knee brace to start active knee flexion and passive knee extension training. After 4-12 weeks of active knee flexion and passive knee extension training, the knee flexion angle gradually increased, reaching $90^{\circ}-120^{\circ}$; the strength of the quadriceps femoris was increased by 
squatting. After 3-6 months, the knee joint had full range of motion, and weight-bearing walking could be continued according to the patient's tolerance. The amount of activity was gradually increased to avoid fatigue and strenuous activities. At 6-9 months, they could gradually resume swimming, rope skipping, and jogging.

For efficacy evaluation, we performed 3.0 T MRI (scanning series: repetition time/echo time 3000/41 ms; field of view: $15 \mathrm{~cm} \times 15 \mathrm{~cm}$; matrix: $240 \times 320$; slice thickness: $3.0 \mathrm{~mm}$; Magnetom, Verio, Siemens, Erlangen, Germany) at 3, 6, and 12 months postoperatively. All the images were imported into the RadiAnt DICOM viewer 5.0 (Medixant, Poznan, Poland), and the data were analyzed in the oblique-sagittal fatsuppressed middle-level imaging. The signal intensity was measured in the four regions: the femoral and the tibial ends of the graft, the quadriceps tendon, and the background (approximately $2 \mathrm{~cm}$ in front of the patellar tendon). The region of interest, which was also the area of the selected sites, was $0.2 \mathrm{~cm}^{2}$ (Fig. 1). The signal intensity of each site was then quantified and used in the SNQ value formula as follows: $S N Q$ value = (signal intensity of $A C L$ graft - signal intensity of quadriceps femoris tendon) / background signal intensity [10]. The mean SNQ values of the femoral and tibial ends of the grafts were taken as the final SNQ values of the grafts. Two physicians participated in the MRI measurements of the grafts. Each physician independently measured the value of each region twice at an interval of 2 weeks to eliminate the memory effect. The average value of the measurements of the two physicians was then used to calculate the SNQ. The intra- and inter-observer reliability was calculated using the results of the measurements. The reliability was high when the intraclass correlation coefficient (ICC) was $>0.75$, medium when the ICC was between 0.4 and 0.75 , and low when the ICC was $<0.4$. All statistical analyses were performed using SPSS software version 20.0 (IBM Corp., Armonk, New York, USA). Differences with p-values of $<0.05$ were considered statistically significant.

The Lysholm score was used to assess the outcomes after the ACL injury. The Lysholm score ranges from 0 to 100 based on eight areas: pain, limping, stair climbing, locking, supporting, swelling, instability, and squatting.

The IKDC Subjective Assessment Form, the standardized international documentation system for knee surgery, consists of 18 questions that emphasize the effects of symptoms, activities of daily living, and physical activity on the knee. The form also assessed total knee function on a 0-100 conversion scale. A score of 100 indicates no symptoms and no restriction on activities of daily living or physical activity.

The Tegner score indicated the sport level of the patients, based on which they are graded on a scale of $0-10$. Grade 0 represented sick leave/disability, and grade 10 represented ability to participate in national or international competitive sports activities. This evaluation table is widely used in the evaluation of the exercise capacity of patients with knee injuries.

SPSS version 20.0 (IBM Corp) was used for statistical analysis. The data are expressed as mean \pm standard deviation. The data sets were compared using repeated-measures analysis of variance and Q 
test. Spearman's correlation coefficient test was used to analyze the correlation between SNQ at 6 months and IKDC, Lysholm, and Tegner scores at 2 years postoperatively.

\section{Results}

Overall, 50 patients met the selection criteria and were included in the final study. There were 28 men and 22 women; the average age was 31.0 years (range, 18-50 years). Surgery was performed on 23 left knees and 27 right knees. The causes of injury were as follows: 31 cases of sports injury, nine cases of traffic accident injury, seven cases of fall-related and heavy object-related injuries, and three other cases. The average time from injury to operation was 36.2 days (range, 5-91 days).

All the incisions healed in the first stage after the operation, and there was no infection or injury to the blood vessels and nerves. All patients were followed up for 24-28 months (mean: 26.6 months). The IKDC, Lysholm, and Tegner scores of the knee joint at 6 months and 2 years postoperatively were significantly higher than those before surgery, and those at 2 years postoperatively were significantly higher than those at 6 months $(p<0.05)($ Table 1$)$.

Table 1

Postoperative knee function scores at each time point

\begin{tabular}{|llll|}
\hline Time & IKDC score & Lysholm score & Tegner score \\
\hline Preoperative & $43.801 \pm 3.163$ & $37.531 \pm 3.836$ & $1.940 \pm 0.738$ \\
\hline 6 Months postoperatively & $61.361 \pm 3.951$ & $58.833 \pm 5.006$ & $2.765 \pm 0.563$ \\
\hline 2 Years postoperatively & $76.539 \pm 6.210$ & $75.442 \pm 7.629$ & $4.721 \pm 0.681$ \\
\hline Statistics & $p<0.000$ & $p<0.000$ & $p<0.001$ \\
\hline IKDC: International Knee Documentation Committee & \\
\hline
\end{tabular}

The intra- and inter-observer ICCs were 0.887 and 0.891 , respectively, indicating that the MRI measurement method had high reliability and could be used to assess graft healing. At 6 months and 2 years postoperatively, the graft SNQ values were $12.516 \pm 6.272$ and $10.795 \pm 6.063$, respectively; the difference was statistically significant $(t=-0.461, p=0.001)$. The SNQ values of 50 patients at 6 months postoperatively were negatively correlated with the Lysholm, IKDC, and Tegner scores at 2 years postoperatively $\left(R^{2}=0.931, p<0.001 ; R^{2}=0.803, p<0.001 ; R^{2}=0.226, p<0.001\right.$, respectively) (Fig. 2$)$.

According to the median SNQ value at 6 months postoperatively, there were 25 cases in group A and 25 cases in group B. The SNQ values of group A at 6 months and 2 years postoperatively were $17.536 \pm$ 4.752 and $13.574 \pm 4.676$, respectively, and those in group $B$ were $8.476 \pm 2.149$ and $6.531 \pm 2.386$, respectively. The knee function scores are shown in Table 2. 
Table 2

SNQ values and knee function scores at different time points in groups $A$ and $B$

\begin{tabular}{|c|c|c|c|c|c|c|c|c|}
\hline & & & A & & & B & & \\
\hline & SNQ & $\begin{array}{l}\text { IKDC } \\
\text { score }\end{array}$ & $\begin{array}{l}\text { Lysholm } \\
\text { score }\end{array}$ & $\begin{array}{l}\text { Tegner } \\
\text { score }\end{array}$ & SNQ & $\begin{array}{l}\text { IKDC } \\
\text { score }\end{array}$ & $\begin{array}{l}\text { Lysholm } \\
\text { score }\end{array}$ & $\begin{array}{l}\text { Tegner } \\
\text { score }\end{array}$ \\
\hline Preoperative & - & $\begin{array}{l}42.520 \\
\pm 4.132\end{array}$ & $\begin{array}{l}39.532 \\
\pm 3.822\end{array}$ & $\begin{array}{l}1.940 \\
\pm \\
0.818\end{array}$ & - & $\begin{array}{l}45.337 \\
\pm 3.182\end{array}$ & $\begin{array}{l}37.382 \\
\pm 3.816\end{array}$ & $\begin{array}{l}1.721 \\
\pm \\
0.646\end{array}$ \\
\hline $\begin{array}{l}6 \text { Months } \\
\text { postoperatively }\end{array}$ & $\begin{array}{l}17.536 \\
\pm 4.752\end{array}$ & $\begin{array}{l}58.323 \\
\pm 4.654\end{array}$ & $\begin{array}{l}56.916 \\
\pm 6.148\end{array}$ & $\begin{array}{l}2.353 \\
\pm \\
0.517\end{array}$ & $\begin{array}{l}8.476 \\
\pm \\
2.149\end{array}$ & $\begin{array}{l}62.236 \\
\pm 3.953\end{array}$ & $\begin{array}{l}60.924 \\
\pm 5.023\end{array}$ & $\begin{array}{l}2.960 \\
\pm \\
0.618\end{array}$ \\
\hline $\begin{array}{l}2 \text { Years } \\
\text { postoperatively }\end{array}$ & $\begin{array}{l}13.574 \\
\pm 4.676\end{array}$ & $\begin{array}{l}73.868 \\
\pm 6.362\end{array}$ & $\begin{array}{l}70.615 \\
\pm 6.179\end{array}$ & $\begin{array}{l}4.643 \\
\pm \\
0.725\end{array}$ & $\begin{array}{l}6.531 \\
\pm \\
2.386\end{array}$ & $\begin{array}{l}81.423 \\
\pm 2.567\end{array}$ & $\begin{array}{l}82.614 \\
\pm 2.436\end{array}$ & $\begin{array}{l}4.776 \\
\pm \\
0.652\end{array}$ \\
\hline \multicolumn{9}{|c|}{ Group A: signal/noise quotient $\geq 12$} \\
\hline \multicolumn{9}{|c|}{ Group B: signal/noise quotient $<12$} \\
\hline \multicolumn{9}{|c|}{ IKDC: International Knee Documentation Committee } \\
\hline \multicolumn{9}{|c|}{ SNQ: signal/noise quotient } \\
\hline
\end{tabular}

In both groups $A$ and $B$, the SNQ value at 6 months postoperatively was negatively correlated with Lysholm, IKDC, and Tegner scores at 2 years postoperatively $(p<0.05)$ (Fig. 3$)$.

\section{Discussion}

The main finding of the study is that the SNQ values at 6 months postoperatively were negatively correlated with the Lysholm, IKDC, and Tegner scores at 2 years postoperatively. Poor graft healing after $A C L$ reconstruction is one of the main reasons for postoperative dysfunction and revision surgery [11]. Based on tissue signal intensity analysis using MRI, Li et al. [12] found that there was a positive correlation between the degree of tendon-bone healing and the clinical function score at 12 months postoperatively, but they did not analyze the correlation between graft maturity and the clinical function score. The MRI signal intensity in a region of interest of the graft after ACL reconstruction has been shown to reflect the water content and graft maturity $[8,13]$. Since the safety and reliability of the ACL reconstruction procedure are good, it has good acceptability among patients. In this study, we used MRI to calculate the SNQ value based on the signal intensity of the region of interest of the graft in order to quantitatively measure the graft maturity after ACL reconstruction. A smaller SNQ value indicates better graft maturity. In previous studies, the graft maturation process after ACL reconstruction was divided into three stages, i.e., early necrosis, revascularization, and graft ligamentization, and the graft has been reported to have a high water content and high SNQ at 6 months postoperatively $[14,15]$. The results of this study show that the graft matures gradually after reconstruction, and the SNQ value at 6 months 
postoperatively is higher than that at 2 years postoperatively; this is similar to the above results and further confirms that the SNQ value can better reflect graft maturity.

This study further evaluated the correlation between the SNQ value and the postoperative knee function score. The results showed that the SNQ value of grafts gradually decreased while the clinical function score gradually increased, and this correlated negatively with each other $(p<0.05)$. In this study, patients were divided into groups according to the median SNQ value at 6 months postoperatively. Further, the SNQ differences were used to reflect the knee function at 2 years postoperatively, and the correlation between SNQ and knee function score was clarified. Therefore, the SNQ value of early postoperative MRI can be used to evaluate the graft maturity after ACL reconstruction and further predict the recovery of knee function. For patients with poor graft maturity (higher SNQ value) in the early postoperative period, a specialized rehabilitation program or other measures to promote graft maturation, such as use of plateletrich plasma [16], autologous fibrin clot [17], and stem cell transplantation [18] should be provided.

The study has some limitations. The sample size of this study was small, and the follow-up time was short. The results of this study need to be confirmed by conducting future studies with a large sample size and long-term follow-up. At the same time, whether different fixation devices of the tibia and femur can affect the graft maturity and function score needs to be further investigated.

\section{Conclusion}

The correlation analysis showed that graft maturity and knee function score gradually improved postoperatively, and the early graft maturity level (SNQ value) can be used as an index to predict the longterm knee function of patients undergoing $A C L$ reconstruction surgery. This can guide surgeons to provide further intervention and treatment for patients with higher SNQ values to improve patient recovery.

\section{Abbreviations}

ACL Anterior cruciate ligament

IKDC International Knee Documentation Committee

MRI Magnetic resonance imaging

SNQ signal/noise quotient

\section{Declarations}

\section{Ethics approval and consent to participate}


This study was conducted in accordance with the principles of the Declaration of Helsinki and was approved by the ethics committee of Affiliated Zhongshan Hospital of Dalian University (No. 2019172). All study participants provided informed consent.

\section{Consent for publication}

All presentations of case reports have consent for publication.

\section{Availability of data and materials}

The data and materials are available from the medical records department of the Affiliated Zhongshan Hospital of Dalian University. The datasets used and analyzed during the study are available from the corresponding author on reasonable request.

\section{Competing interest}

Not applicable

\section{Funding}

The authors report no involvement of any sponsor in the research that could have influenced the outcome of this work.

\section{Authors' contributions}

$\mathrm{CR}$ and GX contributed equally to this work. CR contributed to the design of the study, clinical assessment, analysis, interpretation of data, and revision of the manuscript. GX and ZH contributed to the design of the study, data interpretation, and revision of the manuscript for important intellectual content. XX contributed to the design of the study, data acquisition, and critical revision of the manuscript for important intellectual content. All authors have read and approved the final manuscript.

\section{Acknowledgments}

We would like to acknowledge Editage (www.editage.com) for English language editing.

\section{References}


1. Kochhal N, Thakur R, Gawande V. Incidence of anterior cruciate ligament injury in a rural tertiary care hospital. J Family Med Prim Care. 2019;8:4032-5.

2. Weitz FK, Sillanpaa PJ, Mattila VM. The incidence of paediatric ACL injury is increasing in Finland. Knee Surg Sports Traumatol Arthrosc. 2020;28:363-8.

3. Magnitskaya N, Mouton C, Gokeler A, Nuehrenboerger C, Pape D, Seil R. Younger age and hamstring tendon graft are associated with higher IKDC 2000 and KOOS scores during the first year after ACL reconstruction. Knee Surg Sports Traumatol Arthrosc. 2020;28:823-32.

4. Ouillette R, Edmonds $E$, Chambers $H$, Bastrom T, Pennock A. Outcomes of revision anterior cruciate ligament surgery in adolescents. Am J Sports Med. 2019;47:1346-52.

5. Li Q, Zhang Y, Zhan L, Han Q, Wu M, Zhang N. Correlation analysis of magnetic resonance imagingbased graft maturity and outcomes after anterior cruciate ligament reconstruction using International Knee Documentation Committee Score. Am J Phys Med Rehabil. 2019;98:387-91.

6. Weiler A, Peters G, Maurer J, Unterhauser FN, Südkamp NP. Biomechanical properties and vascularity of an anterior cruciate ligament graft can be predicted by contrast-enhanced magnetic resonance imaging. A two-year study in sheep. Am J Sports Med. 2001;29:751-61.

7. Liu S, Li H, Tao H, Sun Y, Chen S, Chen J. A randomized clinical trial to evaluate attached hamstring anterior cruciate ligament graft maturity with magnetic resonance imaging. Am J Sports Med. 2018;46:1143-9.

8. Zhang Y, Liu S, Chen Q, Hu Y, Sun Y, Chen J. Maturity progression of the entire anterior cruciate ligament graft of insertion-preserved hamstring tendons by 5 years: a prospective randomized controlled study based on magnetic resonance imaging evaluation. Am J Sports Med. 2020;48:2970-7.

9. Li H, Chen J, Li H, Wu Z, Chen S. MRI-based ACL graft maturity does not predict clinical and functional outcomes during the first year after ACL reconstruction. Knee Surg Sports Traumatol Arthrosc. 2017;25:3171-8.

10. Ahn JH, Lee SH, Choi SH, Lim TK. Magnetic resonance imaging evaluation of anterior cruciate ligament reconstruction using quadrupled hamstring tendon autografts: comparison of remnant bundle preservation and standard technique. Am J Sports Med. 2010;38:1768-77.

11. Rahardja R, Zhu M, Love H, Clatworthy MG, Monk AP, Young SW. Factors associated with revision following anterior cruciate ligament reconstruction: a systematic review of registry data. Knee. 2020;27:287-99.

12. Li HY, Li H, Wu ZY, Chen JW, Chen SY. MRI-based tendon bone healing is related to the clinical functional scores at the first year after anterior cruciate ligament reconstruction with hamstring tendon autograft. Knee Surg Sports Traumatol Arthrosc. 2018:26:615-21.

13. Wen Z, Zhang H, Yan W, Mohamed SI, Zhao P, Huang X, et al. Oval femoral tunnel technique is superior to the conventional round femoral tunnel technique using the hamstring tendon in anatomical anterior cruciate ligament reconstruction. Knee Surg Sports Traumatol Arthrosc. 2020;28:2245-54. 
14. Claes S, Verdonk P, Forsyth R, Bellemans J. The "ligamentization" process in anterior cruciate ligament reconstruction: what happens to the human graft? A systematic review of the literature. Am J Sports Med. 2011;39:2476-83.

15. Muller B, Bowman KF, Bedi A. ACL graft healing and biologics. Clin Sports Med. 2013;32:93-109.

16. Mehrabani D, Seghatchian J, Acker JP. Platelet rich plasma in treatment of musculoskeletal pathologies. Transfus Apher Sci. 2019;58:102675.

17. Amini MH, Prieto JF. Biologic and mechanical augmentation in anterior cruciate ligament reconstruction: fibrin clot augmentation of 5-strand hamstring autograft. Arthrosc Tech. 2017;6:e1851-5.

18. Kawakami Y, Takayama K, Matsumoto T, Tang Y, Wang B, Mifune Y, et al. Anterior cruciate ligamentderived stem cells transduced with BMP2 accelerate graft-bone integration after ACL reconstruction. Am J Sports Med. 2017;45:584-97.

\section{Figures}

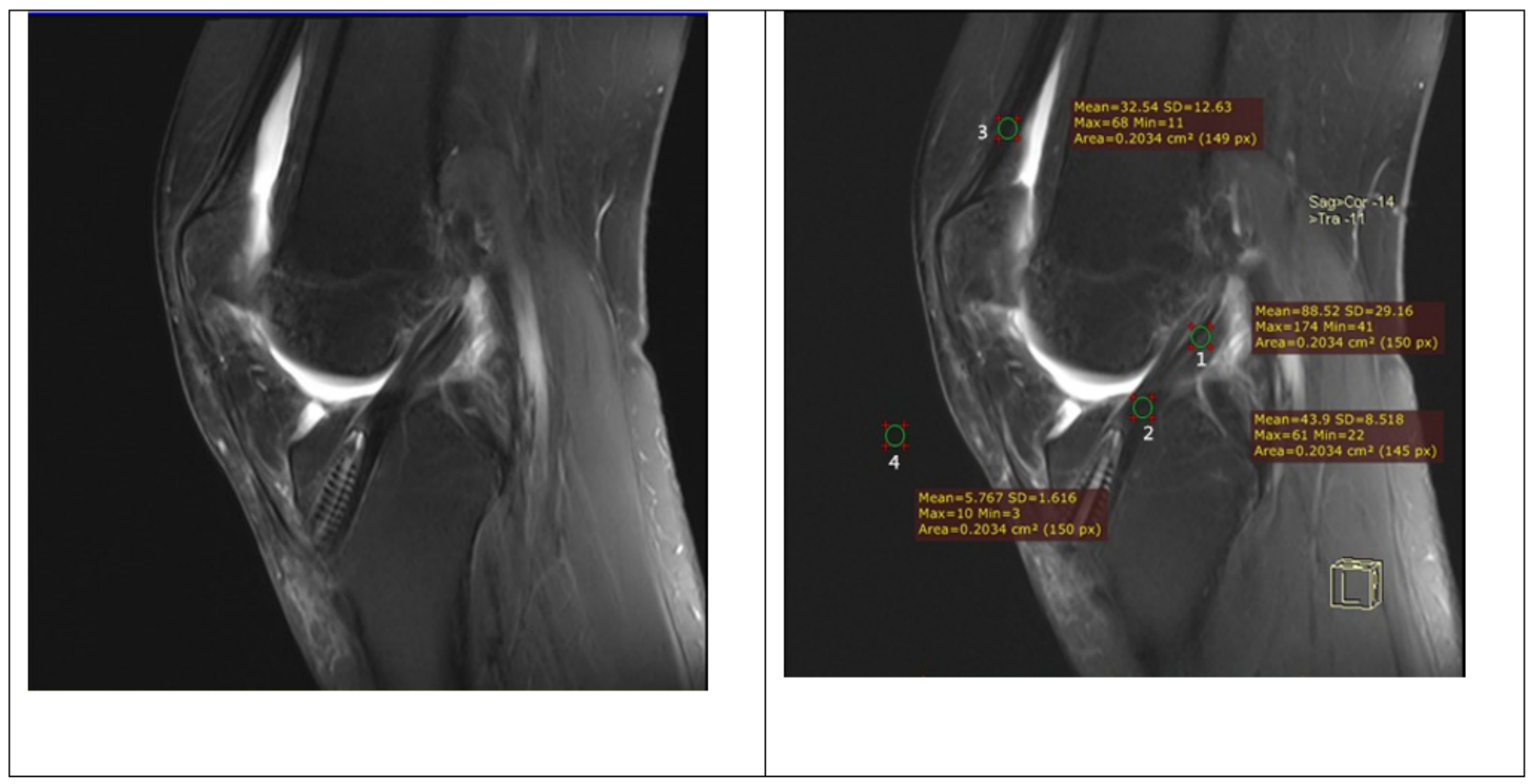

\section{Figure 1}

Magnetic resonance imaging of signal intensity measurement at the graft site in patients who underwent anterior cruciate ligament reconstruction 1: Femoral end of graft, 2: tibial end of graft, 3: quadriceps femoris tendon, 4: background. 


\begin{tabular}{|c|c|c|}
\hline (10) & (10) & $\underset{0}{10}$ \\
\hline $\mathrm{a}$ & b & C \\
\hline
\end{tabular}

Figure 2

Correlation analysis of signal/noise quotient (SNQ) values and knee function scores in 50 patients who underwent anterior cruciate ligament reconstruction a. Correlation between SNQ value and Lysholm scores; b. correlation between SNQ value and IKDC scores; c. correlation between SNQ value and Tegner scores 


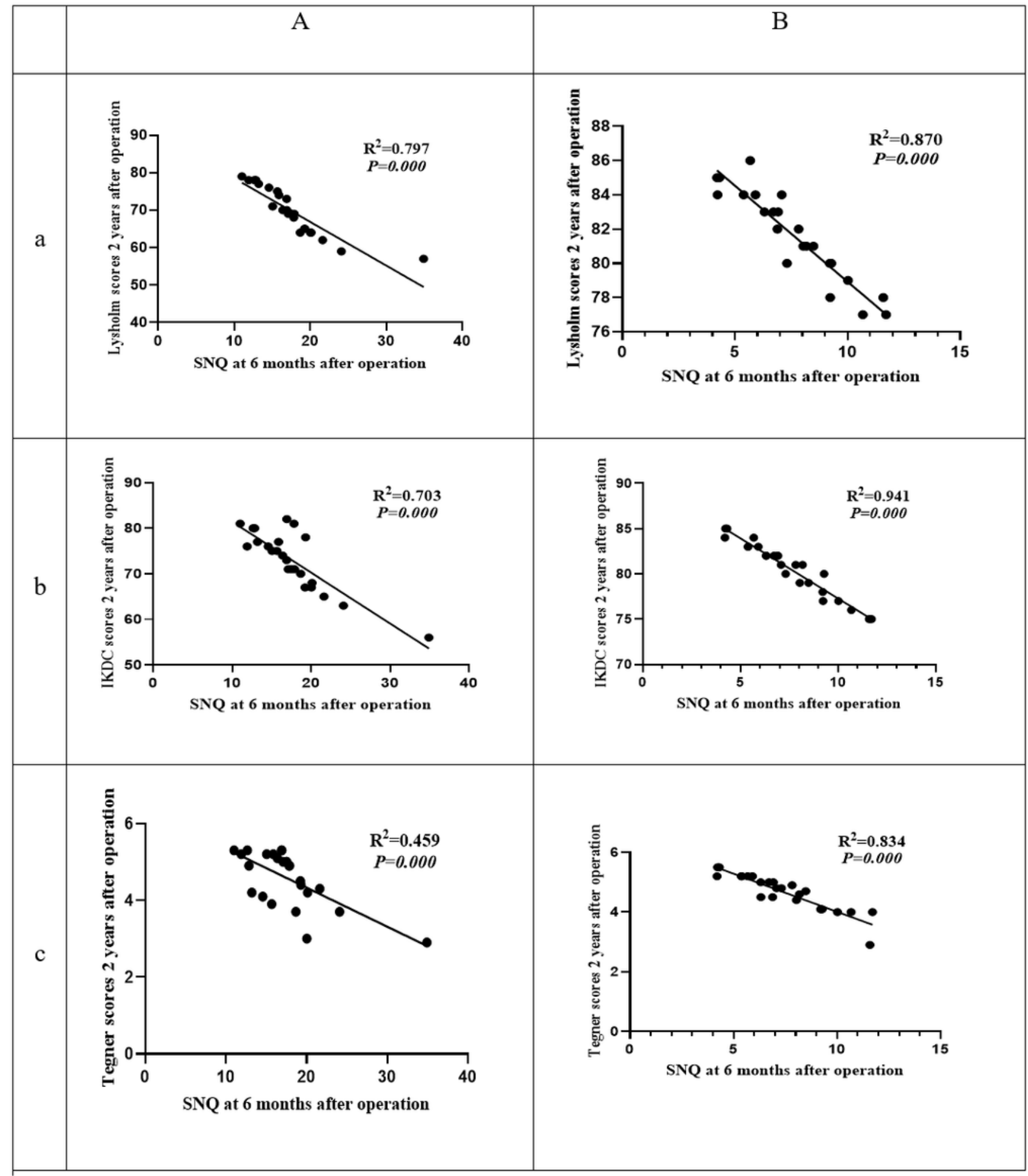

\section{Figure 3}

Correlation analysis of signal/noise quotient (SNQ) values and knee function scores in patients who underwent anterior cruciate ligament reconstruction and were stratified according to the SNQ value Left: group A (SNQ $\geq 12)$ Right: group B (SNQ < 12) a. Correlation between SNQ values and Lysholm scores; $b$. correlation between SNQ values and International Knee Documentation Committee (IKDC) scores; c. correlation between SNQ values and Tegner scores 